\title{
Meta-analysis Shows That Rapid Phenotypic Change in Angiosperms in Response to Environmental Change Is Followed by Stasis
}

\author{
Lucas D. Gorné* and Sandra Díaz
}

Facultad de Ciencias Exactas, Físicas y Naturales, Universidad Nacional de Córdoba, Córdoba, Argentina; and Instituto Multidisciplinario de Biología Vegetal (IMBiV), Consejo Nacional de Investigaciones Científicas y Técnicas (CONICET), Córdoba, Argentina

Submitted September 14, 2018; Accepted July 3, 2019; Electronically published September 27, 2019

Online enhancements: supplemental PDF.

\begin{abstract}
AвSTRACT: The amount and rate of phenotypic change at ecological timescales varies widely, but there has not been a comprehensive quantitative synthesis of the patterns and causes of such variation for plants. Present knowledge is based predominantly on animals, whose differences with plants in the origin of germ cells and the level of modularity (among others) could make it invalid for plants. We synthesized data on contemporary phenotypic responses of angiosperms to environmental change and show that if extinction does not occur, quantitative traits change quickly in the first few years following the environmental novelty and then remain stable. This general pattern is independent from life span, growth form, spatial scale, or the type of trait. Our work shows that high amounts and rates of phenotypic change at contemporary timescales observed in plants are consistent with the pattern of stasis and bounded evolution previously observed over longer time frames. We also found evidence that may contradict some common ideas about phenotypic evolution: (1) the total amount of phenotypic change observed does not differ significantly according to growth form or life span; (2) greater and faster divergence tends to occur between populations connected at the local scale, where gene flow could be intense, rather than between distant populations; and (3) traits closely related to fitness change as much and as fast as other traits.
\end{abstract}

Keywords: plants, phenotypic change, phenotypic evolution, contemporary evolution, haldanes, darwins.

\section{Introduction}

Phenotypic change in quantitative traits within a species or population at timescales between a few months and a few centuries (Kauffman and Levin 1987; Fitch and Ayala 1994) is of critical importance for two main reasons. First, ecolog-

\footnotetext{
* Corresponding author; email: gorneld@gmail.com. ORCIDs: Gorné, https://orcid.org/0000-0003-1940-1755; Díaz, https:/orcid .org/0000-0003-0012-4612.
}

Am. Nat. 2019. Vol. 194, pp. 840-853. (C) 2019 by The University of Chicago. 0003-0147/2019/19406-58735\$15.00. All rights reserved.

DOI: $10.1086 / 705680$ ical timescales are the most relevant given the accelerated environmental changes facing most ecosystems (IPCC 2014, 2018). Second, there is unexplained variation in the rates and patterns of change in quantitative traits across different timescales (summarized by Voje 2016; Voje et al. 2018). Given that the amount and rate of phenotypic change at ecological timescales varies widely (Hendry and Kinnison 1999; Bone and Farres 2001; Crispo et al. 2010; Westley 2011), the critical questions are whether, in the face of environmental change, a population will adapt and whether its natural history and the nature of the environmental change can predict this outcome. Understanding and predicting phenotypic evolution (Lande 1976) in nature still poses a challenge because of the complex interplay among natural selection, mutation, drift, gene flow, and evolutionary constraints (Arnold 1992; Futuyma 2010; Hansen et al. 2011). However, general questions about patterns and processes in contemporary phenotypic evolution can be addressed using broad-scale compilations of observed phenotypic change in the field and laboratory (Hendry and Kinnison 1999; Kinnison and Hendry 2001).

The relationships between phenotypic change and elapsed time are informative of the predominant mode of evolution. Consider three general modes of evolution: (i) directional change, where a quantitative trait changes in a population, generation after generation, always in the same direction; (ii) random change, where the direction of the change in each generation is random; and (iii) stasis, where a value of the trait acts as an attractor (fig. 1). If there is directional change, the rate of evolution is constant across time, so the slope of the log-log relation between elapsed time and rate of change is expected to be zero (Gingerich 1983, 1993, 2009; Hunt 2012). If there is random change or stasis, the rate of change declines with time (Hunt 2012) with a slope expected to be -0.5 for random walk and -1 for stasis (Gingerich 1983 , 1993, 2009). Additionally, the slope of the log-log relation 


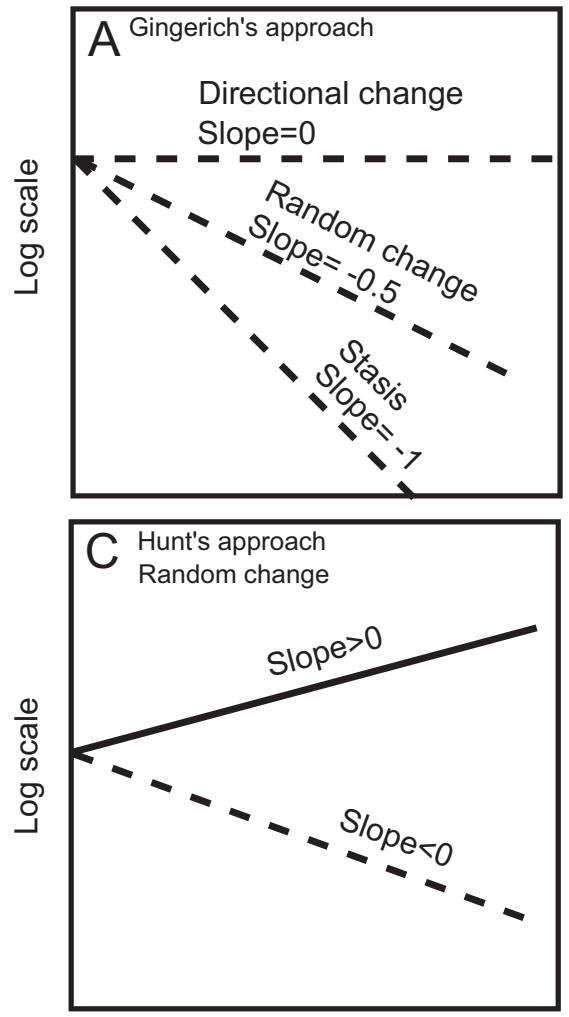

Log (time)
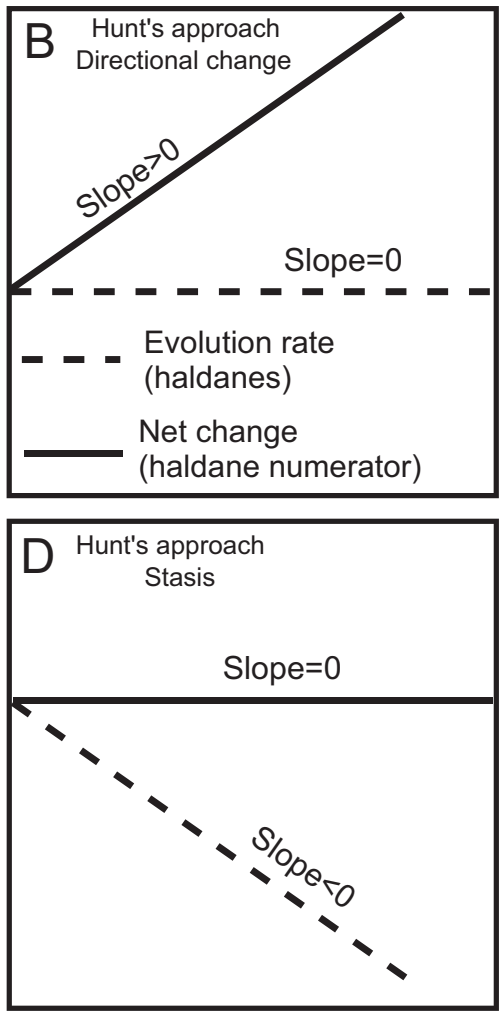

Log (time)

Figure 1: Gingerich's (2009) and Hunt's (2012) approach to the three standard modes of evolution (directional change, random change, stasis). Gingerich's approach analyzes the slope of the log-log relation between the rate of evolution (like haldanes, dashed line) and the elapsed time. If there is directional change, the rate of evolution is constant across time, so the slope is zero. If there is random change or stasis, the rate of change declines with time with a characteristic slope ( -0.5 for random walk and -1 for stasis; $A)$. Hunt's approach analyzes the $\log -\log$ relations of the elapsed time with the mean of the generalized random walk $\left(\mu_{s} ;\right.$ haldanes $\left.\cong \mu_{s} ; \operatorname{LR} \cong \log _{10}\left(\mu_{s}\right)\right)$ and with the divergence $\left(\omega ; \omega \cong\right.$ haldane's numerator; $\left.L G \cong \log _{10}(\omega)\right)$. In a situation of directional change, the slopes of the log-log relation of the elapsed time with the rate of change is expected to be zero and with the amount of change is expected to be positive $(B)$. In a situation of unbiased random walk, the slopes of the log-log relation of the elapsed time with the rate of change is expected to be negative and with the amount of change is expected to be positive $(C)$. In a stasis situation, the slopes of the log-log relation of the elapsed time with the rate of change is expected to be negative and with the amount of change is expected to be zero $(D)$.

between amount of change and elapsed time is expected to be positive for directional or unbiased random walk and zero for stasis (Hunt 2012).

Some recurrent patterns emerge from compilations of contemporary phenotypic change in animals and plants. First, for both animals and plants, rates of change have a lognormal distribution, indicating that small rates of change are much more common than large ones (Bone and Farres 2001; Kinnison and Hendry 2001). This pattern is usually explained by arguing that natural populations are at least partly adapted to their local selective environments but at any particular location the intensity and direction of selection will fluctuate over time, making adaptation an ongoing and everchanging necessity (Hendry et al. 2008). Second, also for both animals and plants, the relation between absolute amount of change and elapsed time is very weak or null (Bone and
Farres 2001; Kinnison and Hendry 2001; Hendry et al. 2008; Crispo et al. 2010). Third, only for animals, the log$\log$ relation between the rate of change and the elapsed time is linear and negative (Gingerich 1983, 1993, 2001, 2009; Hendry and Kinnison 1999; Kinnison and Hendry 2001). Rates may decrease with time if genetic potential is exhausted by directional selection, if a local adaptive peak is approached, or if the direction of change is variable over time (Lynch 1990; Kinnison and Hendry 2001; Grant and Grant 2002). According to Gingerich's approach (fig. 1A), some previous reports are consistent with stasis (Gingerich 2001; Kinnison and Hendry 2001). Hunt's approach (fig. $1 B$ $1 D$ ) has not been included before in analyses of contemporary phenotypic change; however, following his analytical framework, published syntheses are consistent with stasis (Bone and Farres 2001; Kinnison and Hendry 2001; Hendry et al. 
2008; Crispo et al. 2010) or random change (Kinnison and Hendry 2001).

However, in the case of plants, the relation between the rate of change and elapsed time and the frequency distribution of the absolute amount of change are not known. Only two compilations, which summarized 78 observations from 26 species (Bone and Farres 2001) and 212 observations from 13 species (Crispo et al. 2010), have been published to date. In addition, patterns of phenotypic evolution may be taxon dependent (Crispo et al. 2010). Several fundamental biological differences between plants and animals (e.g., modular vs. unitary organisms, sessile vs. mostly motile organisms, absence vs. presence of a germ cell line) raise the question of whether the findings identified mainly for animals are also valid for plants (Jerling 1985). For example, in plants each module is capable of reproduction, and the lack of a germ cell line makes plants more susceptible to and able to propagate somatic mutations.

The absolute amount and rate of phenotypic change can be affected by a number of factors. Rates of phenotypic change do not appear to differ between annuals and perennials, measured either in years (darwins) or standardized by generation time (haldanes; Bone and Farres 2001); however, this was based on data from only three perennial species, all of them herbaceous. In the present study, we expected that annuals would adapt more quickly in absolute time because of their shorter generation time, especially compared with long-lived perennials such as trees or clonal plants. The fact that some traits have more potential to evolve than others can also affect the rate and absolute amount of change (Houle 1992; Hansen et al. 2011). The more complex the trait is (e.g., life-history traits, closely linked to survival, growth, fecundity), the higher its genetic variation, so its evolvability is expected to be greater (Hansen et al. 2011). Life-history traits are also expected to be under strong directional selection (Fisher 1930); as a consequence, we expected them to show higher amounts and rates of phenotypic change. In addition, the absolute amount and rate of change in physiological traits have been found to be higher than those in morphological traits (Bone and Farres 2001), suggesting more evolvability of or greater natural selection on physiological traits.

There has been no systematic attempt to summarize estimates of absolute amounts and rates of phenotypic change in plants since the works of Bone and Farres (2001) and Crispo et al. (2010). Here we analyze the most comprehensive database to date of contemporary phenotypic change in plant quantitative traits (Gorné and Díaz 2017b), which contains observations from field situations as well as commonconditions experiments. We used a combination of a recent meta-analytic approach (Gorné and Díaz 2017a), frameworks of evolution developed within the context of paleontology (Gingerich 2009; Hunt 2012), and insights into factors affecting contemporary phenotypic change (Hendry and Kinnison 1999) to ask, How does phenotypic change unfold in the elapsed time since the triggering environmental novelty? How is phenotypic change affected by spatial scale, the degree of novelty of the environmental change involved, trait variation source, plant growth form, life span, and trait type? And which evolutionary mode-directional change, random change, or stasis - predominates in plant phenotypic change at ecological timescales?

\section{Material and Methods}

Database

Here we applied the methods proposed by Gorné and Díaz (2017a) to a revised version of their database (Gorné and Díaz 2017b, 2019). In brief, this database included studies that provide data on changes in quantitative traits of angiosperms within a known temporal framework ( $<300$ years). The search was performed using Scopus (http://www.scopus .com), up to December 22, 2015 (search strings are in Gorné and Díaz 2017a). The database includes studies that measured intraspecific change in a quantitative trait and that report the elapsed time when the phenotypic change occurred. The studies recorded a single population before and after a change in the environment or compared two (or more) populations by measuring a quantitative trait across two situations, where one of them was a new condition of known age. Studies reporting results from artificial selection or interspecific hybridization were excluded. The environmental changes included expansions of distributional range, soil or air pollution, exposure to herbicides, changes in salinity, $\mathrm{pH}$, climate, disturbance or irrigation regime, and addition or loss of species in the local community. All data available in each study were recorded, including several observations of the same species. These procedures resulted in a database containing 1,716 observations from 128 studies, with changes in populations of 152 species from 34 families, in elapsed times of $<260$ years, and covering a wide range of traits, life span, growth forms, and environmental situations (table 1). Because the rma.mv function ( $\mathrm{R}$ package metafor) cannot deal with a range of effect size weights broader than $10^{7}$, we had to remove two cases with extremely high variance. Because the weight given to any observation is inversely proportional to its variance, the eliminated cases would have had extremely low influence on the results. We retained a final number of 1,714 observations for analysis; no study or species was lost as a result of the two removed observations.

\section{Data Categorization}

All data points were categorized according to biological properties of the study system (life span, growth form, trait type) 
and methodological ones that can modify the patterns observed (moderating variables; table 1). While some studies measure populations directly in the field, others study their descendants under common conditions to assess genetic change (Hendry and Kinnison 1999). Because sometimes epigenetic effects persist under these common-conditions experiments and thus can be confounded with truly genetic effects and because ultimately in all cases phenotypic change in quantitative traits was measured, here we refer to "cisgenerational" versus "transgenerational" studies (instead of phenotypic vs. genetic). Another methodological distinction is between synchronic studies that measure divergence between two or more populations separated in space (also called transversal studies) and allochronic (longitudinal) studies that follow a population over time and measure its evolution (Kinnison and Hendry 2001). A third methodological consideration is one of the spatial scale of work, which ranges from populations growing a few meters apart to those growing on different continents. Also, we accounted for degree of novelty in the environmental change by categorizing each case as "novel" (i.e., the environmental change has a defined starting point and phenotypic change was measured from this point) or "ongoing" (i.e., the environmental change has been progressing for awhile and the phenotypic change was measured in a temporal window within this process). Finally, older studies have been found to report more intense selection gradients, introducing a bias toward larger absolute amounts and rates of change (Kinnison and Hendry 2001).

\section{Effect Sizes}

The effect sizes published in the database (Gorné and Díaz $2017 b$ ) were the standardized mean difference Hedges $g$ (Hedges 1981, 1982; supplemental PDF, available online), a rate of change $(h)$ that is the Hedges $g$ over the elapsed time in years, and the log transformation of both of them, that is, LG and LR (Gorné and Díaz 2017a; supplemental PDF). The units of these last two effect sizes (LG and LR) are $\log _{10}(\mathrm{SD})$ and $\log _{10}\left(\mathrm{SD} \cdot \mathrm{year}^{-1}\right)$, respectively. We used both of them to analyze the effect of the moderating variables on the absolute amount and rates of change as well as on the pattern of change in the framework proposed by Gingerich (2009) and Hunt's approach (Hunt 2006, 2012). The methods to compute the effect sizes, explained in detail in Gorné and Díaz (2017a), followed standard meta-analytic procedures (Hedges 1982; DeCoster 2004; Cooper et al. 2009).

The fundamental effect size here, standardized mean difference, fits adequately with the magnitude we intended to measure - that is, the change in a quantitative trait between two samples - and it is equal to the haldane numerator, which is a standard rate of evolution (Haldane 1949; Gingerich 1993). Additionally, the haldane numerator is a variance- scaled metric (i.e., expresses the difference in units of standard deviations) and is equal to the response $(R)$ to selection $(S)$ according to the breeder's equation (Lush 1947; Gingerich 2009).

In addition, we upgraded the Díaz and Gorné (2017b) database, computing the response ratio effect size (log RR; Hedges et al. 1999) whenever possible. The response ratio is a mean-scaled metric equal to the darwins numerator (Haldane 1949). From log RR we computed a rate of change similar to darwins ( $\mathrm{dw}$ ); time is expressed as years instead of millions of years (supplemental PDF).

\section{Model Construction and Selection Criteria}

To assess the effects of the different moderating variables on the amount (LG and $|\log \mathrm{RR}|$ ) and rate of change (LR and $\left.\log _{10}(|d w|)\right)$, we performed a model selection procedure (using the maximum likelihood method and the Akaike information criterion). The models were then refitted by the restricted maximum likelihood method to estimate the coefficients.

In the model selection procedure, we proceeded by steps: we first tested methodological moderating variables (spatial scale, design, publication year, elapsed time, environmental change, trait variation source; table 1). Because the effect of local scale was different from those of regional and continental scales but the latter two did not significantly differ from each other, we replaced the original scale variable with a second one that split scale into local versus nonlocal (the latter including regional plus continental). Second, we added the biological moderating variables. Because growth form and life span are not independent (there is no woody short-lived species), we combined both in a factor with the following levels: herbaceous short lived, herbaceous long lived, and woody. We alternatively tested a moderating variable distinguishing woody from herbaceous species. Finally, we added the trait type moderating variable to the model. The models fitted in each stage are shown in table S1 (tables S1, S2 are available online). From the fitted models for LG and LR, we computed the intrinsic rate of change, that is, the amount of change expected in a unit of time (Gingerich 2009; Hunt 2012).

The data set presented two sources of nonindependence. First, multiple effect sizes had been taken from each study (paperID). Second, multiple effect sizes had been taken from each species (spID). Additionally, we tested for phylogenetic signal in the residuals of the fitted models (Revell 2010) using the R package phylosignal (Keck et al. 2016) and the phylogenetic tree generated by Phylomatic (ver. 3; Campbell and Donoghue 2005; http://phylodiversity.net/phylomatic/) based on the megatree published by Zanne et al. (2014). To take into account these sources of nonindependence, we used the rma.mv function from the $\mathrm{R}$ package metafor (Viechtbauer 
Table 1: Moderators used to categorize the data points considered in the meta-analysis

\begin{tabular}{|c|c|c|c|c|c|}
\hline \multirow[b]{2}{*}{ Moderating variable, level } & \multicolumn{2}{|c|}{ Variance scaled } & \multicolumn{2}{|c|}{ Mean scaled } & \multirow[b]{2}{*}{ Description (nonexhaustive) } \\
\hline & No. spp. & No. obs. & No. spp. & No. obs. & \\
\hline \multicolumn{6}{|l|}{ Spacial scale $(\mathrm{S})$ : } \\
\hline Local (1) & 48 & 584 & 34 & 444 & Comparison between populations separated by $<10 \mathrm{~km}$ \\
\hline Regional (r) & 61 & 321 & 8 & 73 & $\begin{array}{l}\text { Comparison between populations separated by }>10 \mathrm{~km} \\
\text { but occurring in the same continent }\end{array}$ \\
\hline Continental (c) & 57 & 809 & 47 & 634 & Comparison between populations in different continents \\
\hline \multicolumn{6}{|l|}{ Design (D): } \\
\hline Allochronic (a) & 64 & 340 & 10 & 151 & $\begin{array}{l}\text { Longitudinal studies, that is, following a population } \\
\text { across time }\end{array}$ \\
\hline Synchronic (s) & 100 & 1,374 & 79 & 1,000 & $\begin{array}{l}\text { Transversal studies, that is, looking at the divergence } \\
\text { between populations in time }\end{array}$ \\
\hline \multicolumn{6}{|l|}{ Age of publication $(\mathrm{Y})$ : } \\
\hline None & 152 & 1,714 & 85 & 1,151 & $\begin{array}{l}\text { Year of publication minus year of oldest publication in } \\
\text { database (1970) }\end{array}$ \\
\hline \multicolumn{6}{|l|}{ Elapsed time $(\mathrm{t})$ : } \\
\hline None & 152 & 1,714 & 85 & 1,151 & $\begin{array}{l}\text { Time elapsed between the onset of the environmental } \\
\text { novelty and the measurement }\end{array}$ \\
\hline \multicolumn{6}{|l|}{ Environmental change (ec): } \\
\hline Novel (n) & 143 & 1,593 & 82 & 1,061 & $\begin{array}{l}\text { The environmental change has a defined starting point } \\
\text { and phenotypic change was measured from this point }\end{array}$ \\
\hline Ongoing (o) & 13 & 121 & 3 & 90 & $\begin{array}{l}\text { The environmental change has been progressing for a } \\
\text { while and the phenotypic change was measured in a } \\
\text { temporal window within this process }\end{array}$ \\
\hline \multicolumn{6}{|l|}{ Trait variation source (vs): } \\
\hline Cisgenerational (cis) & 102 & 425 & 43 & 196 & $\begin{array}{l}\text { Study considers the variability present in the field, not } \\
\text { allowing phenotypic plasticity to be distinguished } \\
\text { from heritable variability }\end{array}$ \\
\hline Transgenerational (trans) & 79 & 1,289 & 56 & 955 & $\begin{array}{l}\text { Study considers the heritable change, that is, common } \\
\text { garden or reciprocal transplant }\end{array}$ \\
\hline \multicolumn{6}{|l|}{ Growth form (gF): } \\
\hline $\begin{array}{l}\text { Nongraminoid herb (herb } \\
\text { nongram) }\end{array}$ & 103 & 1,206 & 57 & 798 & $\begin{array}{l}\text { Herbaceous plants with no grasslike appearance, mostly } \\
\text { nonmonocotiledoneus }\end{array}$ \\
\hline Graminoid herb (gram) & 37 & 333 & 21 & 235 & $\begin{array}{l}\text { Herbaceous plants in the Poaceae and other families } \\
\text { with a grasslike appearance, all monocotiledoneus }\end{array}$ \\
\hline Woody & 12 & 175 & 7 & 118 & Trees, shrubs, woody vines \\
\hline \multicolumn{6}{|l|}{ Life span (l): } \\
\hline Annual (ann) & 46 & 318 & 19 & 204 & Natural life span $\leq 1$ year \\
\hline Perennial (per) & 76 & 1,053 & 46 & 692 & Natural life span $>2$ years \\
\hline Intermediate (int) & 30 & 343 & 20 & 255 & $\begin{array}{l}\text { Biennial plants and species described as annuals or } \\
\text { short-living perennials depending on the context }\end{array}$ \\
\hline \multicolumn{6}{|l|}{ Trait type (Tr): } \\
\hline Morphological (m) & 80 & 384 & 31 & 173 & $\begin{array}{l}\text { Leaf area, specific leaf area, leaf length and width, leaf } \\
\text { shape, leaf number of adult plant, height of adult } \\
\text { plant, number of shoots or stems, length of shoots, } \\
\text { symmetry, root diameter, root-to-shoot ratio, root } \\
\text { architecture, trichome density, size of floral and fruit } \\
\text { parts, petiole and stipules }\end{array}$ \\
\hline Physiological (f) & 39 & 240 & 24 & 183 & $\begin{array}{l}\text { Photosynthetic and metabolic parameters, concentration } \\
\text { of several substances in plant tissues, and tolerance } \\
\text { to pollution, salinity, drought, or biomass removal }\end{array}$ \\
\hline Life-history traits (h) & 73 & 478 & 51 & 365 & $\begin{array}{l}\text { Various ways to express individual growth rate (in- } \\
\text { crease in biomass, height, length, number of leaf or } \\
\text { tillers in a elapsed time); increase in number or size } \\
\text { of seeds, flowers, or fruits; age or size at maturity; } \\
\text { seed viability; survival; emergence time of seedlings; } \\
\text { offspring dispersal; pollen quantity and viability }\end{array}$ \\
\hline
\end{tabular}


Table 1 (Continued)

\begin{tabular}{|c|c|c|c|c|c|}
\hline \multirow[b]{2}{*}{ Moderating variable, level } & \multicolumn{2}{|c|}{ Variance scaled } & \multicolumn{2}{|c|}{ Mean scaled } & \multirow[b]{2}{*}{ Description (nonexhaustive) } \\
\hline & No. spp. & No. obs. & No. spp. & No. obs. & \\
\hline Biotic relations (r) & 46 & 301 & 31 & 189 & $\begin{array}{l}\text { Any variable taken as response to a treatment that } \\
\text { involves a direct realized biotic relation, such as } \\
\text { herbivory, interspecific or intraspecific competition, } \\
\text { allelopathy, mycorrhizal or rhizobial colonization, } \\
\text { seed or seedlings predation, parasitism }\end{array}$ \\
\hline Phenology (p) & 36 & 77 & 29 & 61 & Flowering, fruiting, leafing time \\
\hline Phenotypic plasticity $(\mathrm{pl})$ & 13 & 234 & 9 & 180 & $\begin{array}{l}\text { Considered as a trait in itself, that is, the change in } \\
\text { plasticity independent of the nature of the plastic } \\
\text { trait }\end{array}$ \\
\hline
\end{tabular}

Note: For each level of these variables, the number of observations (obs.) and the number of species (spp.) are specified for the variance-scaled measurements and the mean-scaled measurements.

2010) to perform multilevel (hierarchical) models, where paperID and spID were included as random factors. No phylogenetic signal was found in the residuals of any model.

\section{Sensitivity Analysis}

To evaluate the sensitivity of the analysis to potentially influential cases, we randomly sampled the data set 100 times. Each time we sampled $70 \%$ of the data (all cases were removed at least once) and fitted models 2 h.b, 3c.d, and 4 f.b (table S1). Then we compared the estimated coefficients and the confidence intervals (CIs) of the set of iterations with the fitted model with all data. The weight of each observation is the same for LG as well as LR, so that it is not necessary to perform the same procedure with model 1h.b.

In this database it is not possible to test for publication bias because all available procedures to detect and assess publication bias assume that, given an effect, there is a symmetrical distribution of outcomes surrounding it, and in our case the typical distributions of LG, $|\log R R|, L R$, and $\log _{10}(|d w|)$ are left skewed (Gorné and Díaz 2017a). This is because the expected distribution of a random collection of amounts and rates of change is a normal distribution with mean equal to zero; therefore, its absolute value is a folded normal distribution (Tsagris et al. 2014). The log transformation of such a folded normal distribution is expected to be left skewed (not symmetrical).

\section{Calculation of Instantaneous Rate of Transgenerational Phenotypic Change}

The $h$ estimated for each elapsed time $\left(h_{t}\right)$ was the average annual change observed in that period and not an instantaneous rate. To estimate an instantaneous rate, we created a new variable, $h_{t}^{\prime}$, which excludes the previously accumulated change $\left(h_{t}^{\prime}=h_{t} \cdot t-h_{t-1} \cdot(t-1)\right)$. From the selected model (2h.a; table S1), we estimated $h_{t}\left(h=10^{\mathrm{LR}}\right)$ for transgenerational phenotypic change at the local scale and in novel environmental change for an average plant type. From that, we computed $h_{t}^{\prime}$ and its CI. The $95 \%$ CI for each time $(t)$ was computed as $h_{t}^{\prime} \pm 1.96 \cdot \mathrm{SE}\left(h_{t}^{\prime}\right)$, where $\mathrm{SE}\left(h_{t}^{\prime}\right)$ is the standard error of $h_{t}^{\prime}$. SE $\left(h_{t}^{\prime}\right)$ was computed iteratively for each time interval, from one to the maximum elapsed time in the database, according to the error propagation theory (Bevington and Robinson 2003). Because our data represent a random sample of rates of change in different times, not temporal series, we assumed uncorrelated errors and computed $\operatorname{SE}\left(h_{t}^{\prime}\right)$ as $\operatorname{SE}^{2}\left(h_{t}^{\prime}\right)=\operatorname{SE}^{2}\left(h_{t}\right)+\operatorname{SE}^{2}\left(h_{t-1}\right)$, where $\operatorname{SE}\left(h_{x}\right)$ is the standard error of $h$ at time $x$. Because $h=10^{\mathrm{LR}}, h=$ $\left(e^{\ln 10}\right)^{\mathrm{LR}}$ and $\mathrm{SE}\left(h_{t}\right)=h_{t} \cdot \ln (10) \mathrm{SE} \cdot\left(\mathrm{LR}_{t}\right)$, where $\mathrm{SE}\left(\mathrm{LR}_{t}\right)$ is the standard error of $\mathrm{LR}_{t}$, which is obtained from the metaregression.

We also estimated the instantaneous rate of transgenerational phenotypic change for the subset of allochronic (longitudinal) data on annual plants. Because in this case $h_{t}$ matches haldanes (the generational rate of change), we know that in this subset of the data evolution and divergence are not confounded. All analyses were performed in R (R Core Team 2018).

\section{Results \\ Description of the Database}

The observed amounts of absolute change varied widely, from 0.00438 to 26.45 SDs and from 0.001 to 5.26 times. These upper limits correspond to the transgenerational phenotypic change in Senecio inaequidens and Centaurea diffusa, respectively (both perennial forbs in the Asteraceae family). The overall amount of change was 1.2000 SDs (95\% CI, 1.0193-1.4132) and 0.2876 times (95\% CI, 0.2150 $0.3602)$. The rates of change also showed much variation, from $1.89 \times 10^{-5}$ to $8.45 \mathrm{SD} \cdot$ year $^{-1}$ and from $9.23 \times 10^{-6}$ 
to 2.69 times $\cdot$ year $^{-1}$. These upper limits both correspond to the transgenerational phenotypic change in Lolium rigidum (Poaceae). The overall rate of change was $0.0244 \mathrm{SD} \cdot$ year $^{-1}$ (95\% CI, 0.0180-0.0329) and 0.0072 times $\cdot$ year $^{-1}(95 \%$ CI, 0.0051-0.0102).

There were numerous situations in which particular traits of particular species changed very little and only few situations in which either the amount or the rate of change was large. Across the whole database, amounts $(g)$ and rates $(h)$ of change were approximately lognormally distributed (figs. S1, S2; figs. S1-S7 are available online). This was not an artifact resulting from a biased distribution of elapsed times: even the frequency distributions for particular elapsed times did not statistically depart from lognormal (e.g., times $1-10$, which had the greatest average rate of change, or times 100 and 200, which had the greatest frequency of data). Because distributions of the LG and LR effect sizes are expected to be left skewed, the observed symmetry may be due to a less than expected frequency of very low values, suggesting a publication bias against zero change. The same pattern was observed in the mean-scaled rate of change (dw; fig. S3).

To assess the robustness of the results, we performed a sensitivity analysis. For variance-scaled rate of change, the analysis showed that the final selected model (2h.b) was robust to random data subsampling (fig. S4). The final selected models for mean-scaled amount (3c.d) and rate (4f.b) of change were far less robust (figs. S5, S6, respectively). However, even in this case some patterns were robust, as we state below.

\section{Patterns of Phenotypic Change} as a Function of Elapsed Time

In the analysis of the amount of change (LG) and the rate of change (LR), we tested the interaction between the elapsed time ( $\log _{10}$ transformed) and trait variation source, design, environmental change, growth form, and life span (table S1). The final selected model indicates that there are interactions between the elapsed time and trait variation source as well as between elapsed time and growth form (woody vs. herbaceous). Figure 2 shows, for the whole database, both the amount of change (LG) and the rate of change (LR) as functions of the elapsed time and the metaregression lines. The slopes of such metaregression lines and their CIs are shown in figure 3 , on average (fig. $3 A$ ) and by group (fig. $3 B-3 G$ ).

The patterns of change according to Gingerich's and Hunt's approaches can be obtained from figure 3 . The average pattern of change is consistent with stasis according to both analytical frameworks (fig. 3A). However, when the effect of trait variation source is considered, the stasis pattern remains for the transgenerational phenotypic change only (fig. 3C). Cisgenerational phenotypic change, in contrast, shows a pattern where the LR versus $t$ slope is more negative than -1

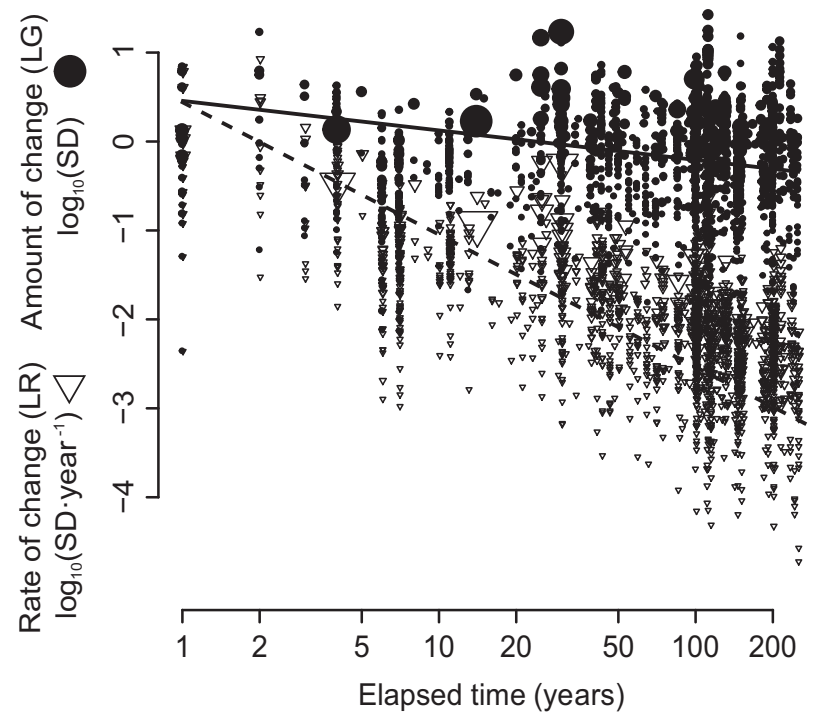

Figure 2: Overview of the results. Shown is the relationship between elapsed time and the amount of change (LG; filled circles) and between elapsed time and the rate of change (LR; open triangles). The size of the symbols represents the weight of each observation in the analysis. Lines represent the average relationship between LG and elapsed time (solid line; table S1, model $1 \mathrm{~h} . \mathrm{a}$ ) as well as between LR and elapsed time (dashed line; table S1, model 2h.a). See table S2 for the full list of original references in the data set.

and the LG versus $t$ slope is significantly negative (fig. $3 B$ ). This pattern is different from the three standard modes (fig. 1) because the amount of change decreases with the elapsed time. As we show later, the intrinsic rate of cisgenerational phenotypic change is substantial and tends to be greater than that of the transgenerational one. However, such cisgenerational phenotypic change does not accumulate through time; rather, it diminishes. We refer to this latter pattern as retraction and we discuss some possible causes below. When cisgenerational phenotypic change in woody and herbaceous species are computed separately, the same retraction pattern is observed for each of them (fig. $3 D, 3 F$ ). The pattern of transgenerational phenotypic change differs between woody and herbaceous species. Woody species show a pattern no different from stasis but also show wide variability and a tendency to retraction (fig. 3G). Herbaceous species, on the other hand, show an intermediate pattern between stasis and unbiased random walk (fig. $3 E$ ). The analysis of mean-scaled amount of change $(|\log R R|)$ shows no interaction between elapsed time and longevity or growth form (table S1); moreover, it does not show any robust effect of time on the amount of change (fig. S5). The analysis of the mean-scaled rate of change $\left(\log _{10}(|d w|)\right)$ showed an effect of elapsed time with a slope close to stasis that was both robust and independent from any other variable in the system (fig. S6). 


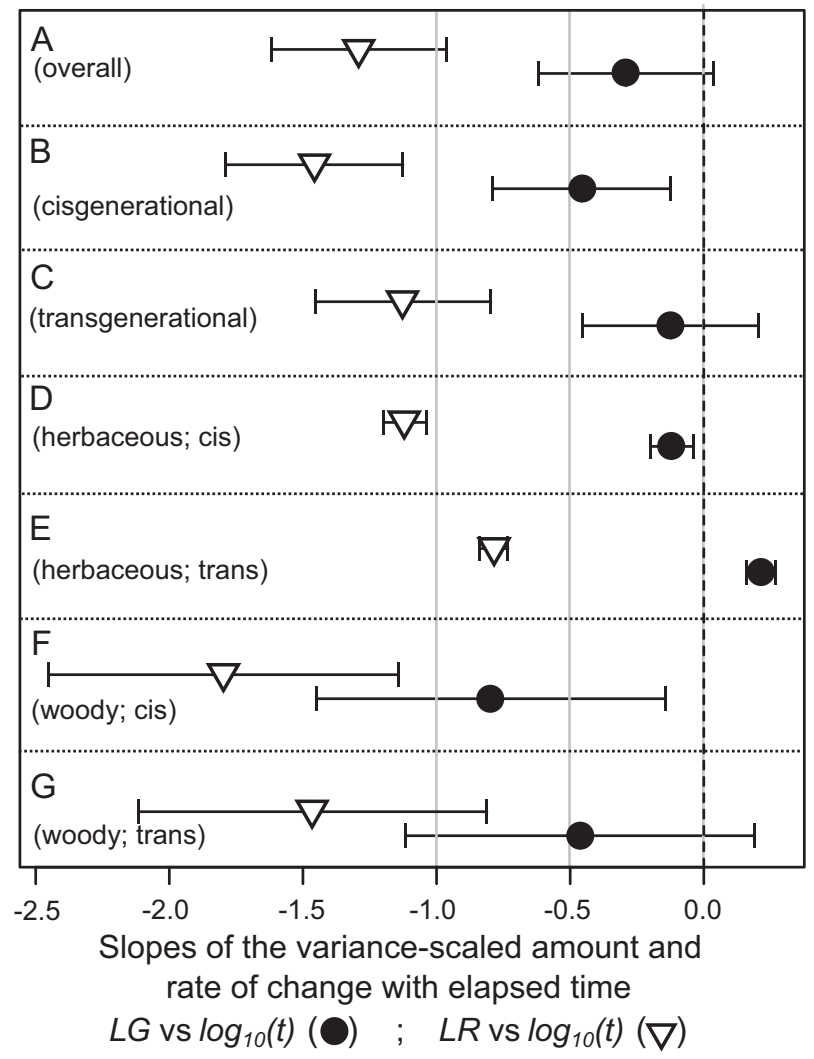

Figure 3: Slopes of the relations between elapsed time ( $\log _{10}$ transformed) and the amount of change (LG; filled circles) and between elapsed time and the rate of change (LR; open triangles) for different data subgroups. Shown are estimates and $95 \%$ confidence intervals for the whole database $(A)$; all plants, cisgenerational phenotypic change $(B)$; all plants, transgenerational phenotypic change $(C)$; herbaceous plants, cisgenerational phenotypic change $(D)$; herbaceous plants, transgenerational phenotypic change $(E)$; woody plants, cisgenerational phenotypic change $(F)$; and woody plants, transgenerational phenotypic change $(G)$. Applying Gingerich's approach, an LR versus $t$ slope of 0 indicates directional change, a slope of -0.5 indicates unbiased random walk, and a slope of -1 indicates stasis. According to Hunt's approach, an LR versus $t$ slope of 0 and an LG versus $t$ slope above 0 indicate directional change; an LR versus $t$ slope below 0 and an LG versus $t$ slope above 0 correspond to unbiased random walk; an LR versus $t$ slope below 0 and an LG vs. $t$ slope of 0 indicate stasis. The slopes estimated from models 1 h.a and 2 h.a are detailed in table S1.

Observed patterns of phenotypic change are consistent with an evolutionary process dominated by stasis; that is, the most frequent rate of change over the whole time interval considered is zero. This does not mean lack of change or homogenous pace of change at different elapsed times. Indeed, the instantaneous rate of transgenerational phenotypic change $\left(h^{\prime}\right)$, estimated jointly for all life spans, growth forms, and trait types, decreased sharply with time (fig. 4). Virtually all of the change occurs in the first few years and then falls to zero. The equivalent analysis for annual plants in allochronic (longitudinal) designs and novel environmental change shows the same pattern (fig. S7).

\section{Intrinsic Rate of Change}

The overall intrinsic rate of change is $3.36 \mathrm{SDs}$ (95\% CI, $0.76-14.85$; gray symbol in fig. $5 \mathrm{~A}$ ). In this case, the intrinsic rate of cisgenerational phenotypic change tends to be greater than the transgenerational one (gray symbols in fig. $5 B$ ), both for woody and for herbaceous plants (gray symbols in fig. 5C, $5 D$ ). While transgenerational phenotypic change involves genetic and epigenetic change, the cisgenerational phenotypic change involves genetic, epigenetic, and phenotypic plasticity, so the difference between these two is due to the effect of phenotypic plasticity. Woody plants show higher values of intrinsic rate of change but with wider variation (gray symbols in fig. 5D). The average amount of change is similar for woody (black symbols in fig. 5D) and herbaceous (black symbols in fig. 5C) plants.

\section{Additive Effects}

Spatial scale, the degree of novelty in the environmental change, and trait type all have similar additive effects in the variance-scaled amount (LG) and rate (LR) of change. The change at local scale (fig. 6) is greater than that at regional or continental scale (dashed line in fig. 6). When the environmental change is ongoing (fig. 6) rather than being

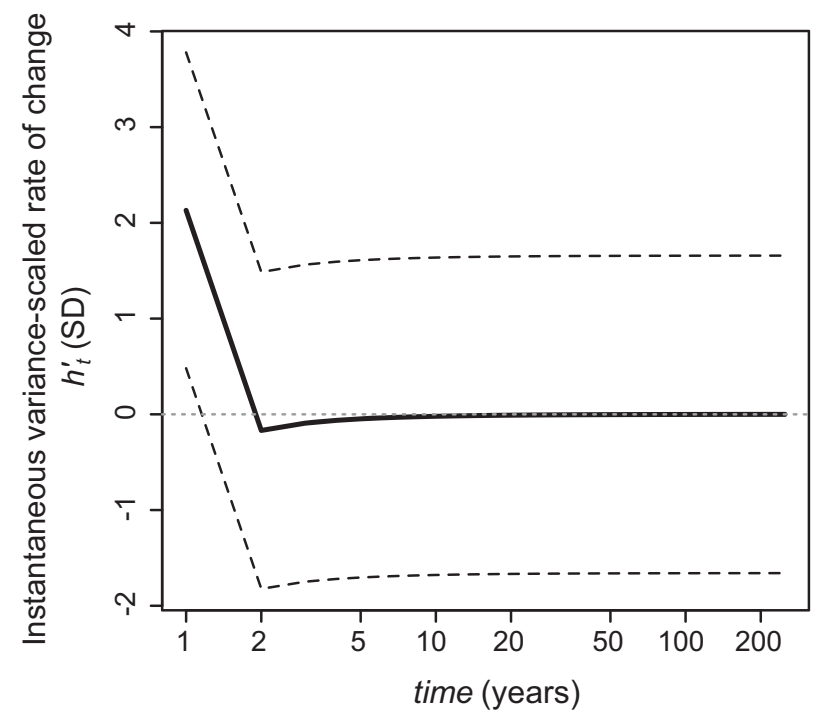

Figure 4: Instantaneous rate of transgenerational phenotypic change $\left(h^{\prime}\right)$ following a novel environmental change for an average plant and trait type at local scale, according to model $2 \mathrm{~h} . \mathrm{a}$ (detailed in table S1). The solid line indicates the mean, the dashed lines indicate the 95\% confidence interval of $h^{\prime}$, and the dotted gray line indicates zero instantaneous rate of change. 


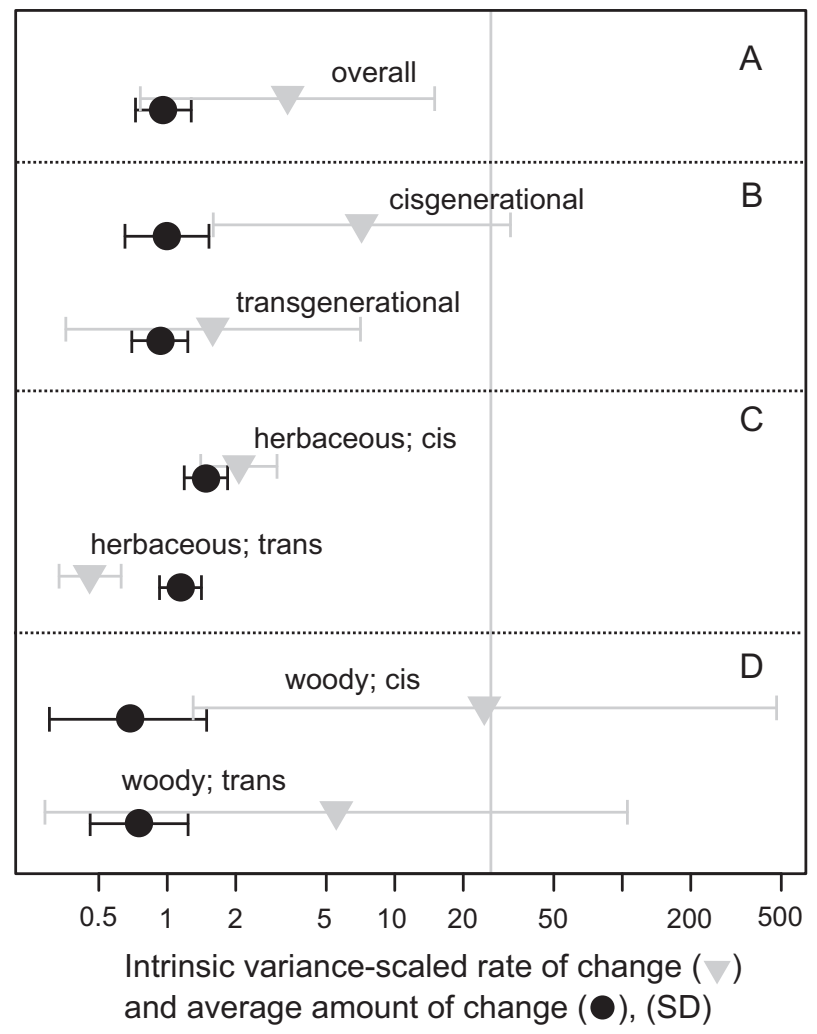

Figure 5: Average amount of change (black circles) and intrinsic rate of change (gray triangles) for the whole database $(A)$; all plants, split by trait variation source $(B)$; herbaceous plants, split by trait variation source $(C)$; and woody plants, split by trait variation source $(D)$. Estimates and $95 \%$ confidence intervals according to models 1 h.a and 10 are detailed in table S1. The gray vertical line is the maximum observed value of the amount of change (Hedge's $g$ ) in the database.

novel (dashed line in fig. 6), the phenotypic change is smaller. The trait type that experiences the least change is phenotypic plasticity (fig. 6), whereas phenological traits are the most labile (fig. 6). Morphological traits (fig. 6) change more than plasticity but less than the other traits. Life-history traits (fig. 6), physiological traits (dashed line in fig. 6), and biotic relations traits (fig. 6) change a similar amount. Design and age of publication were not retained as significant moderating variables by the model selection procedure.

For the mean-scaled amount of change ( $\log R R \mid)$, synchronic (transversal) studies ( $\mathrm{D}=\mathrm{s}$ in fig. S5) robustly show more change than allochronic (longitudinal) ones (dashed line in fig. S5). Woody plants (woody=yes in fig. S5) robustly show less change than herbaceous ones (dashed line in fig. S5). We found the following robust patterns: plasticity traits $(\mathrm{Tr}=\mathrm{pl}$ in fig. S5) change the most; life-history $(\mathrm{Tr}=\mathrm{h}$ in fig. S5), morphological ( $\mathrm{Tr}=\mathrm{m}$ in fig. S5), phenological ( $\mathrm{Tr}=\mathrm{p}$ in fig. S5), and physiological (dashed line in fig. S5) traits change a similar amount; and biotic relations traits ( $\mathrm{Tr}=\mathrm{r}$ in fig. S5) seem to be very sensitive to random data subsampling. The mean-scaled rate of change $\left(\log _{10}(|\mathrm{dw}|)\right)$ robustly tends to be faster at local scale (S2 =l in fig. S6) than at broader scales (dashed line in fig. S6) and tends to be slower in woody species (woody=yes in fig. S6) than in herbaceous ones (dashed line in fig. S6). Regarding the mean-scaled rate of change in the different trait types, we found robust effects only for phenological and plasticity traits, which tend to be the fastest $(\mathrm{Tr}=\mathrm{pl}$ and $\mathrm{Tr}=\mathrm{p}$ in fig. S6).

\section{Discussion}

Our findings show that in response to environmental novelty, quantitative traits of angiosperms change quickly in the first few years and then remain stable. This general pattern is supported by the variance-scaled measure of change, the mean-scaled measure of change, and the instantaneous rate of change $\left(h^{\prime}\right)$. This pattern may also explain the lack of relation between phenotypic change and elapsed time found in previous reports (Bone and Farres 2001; Hendry

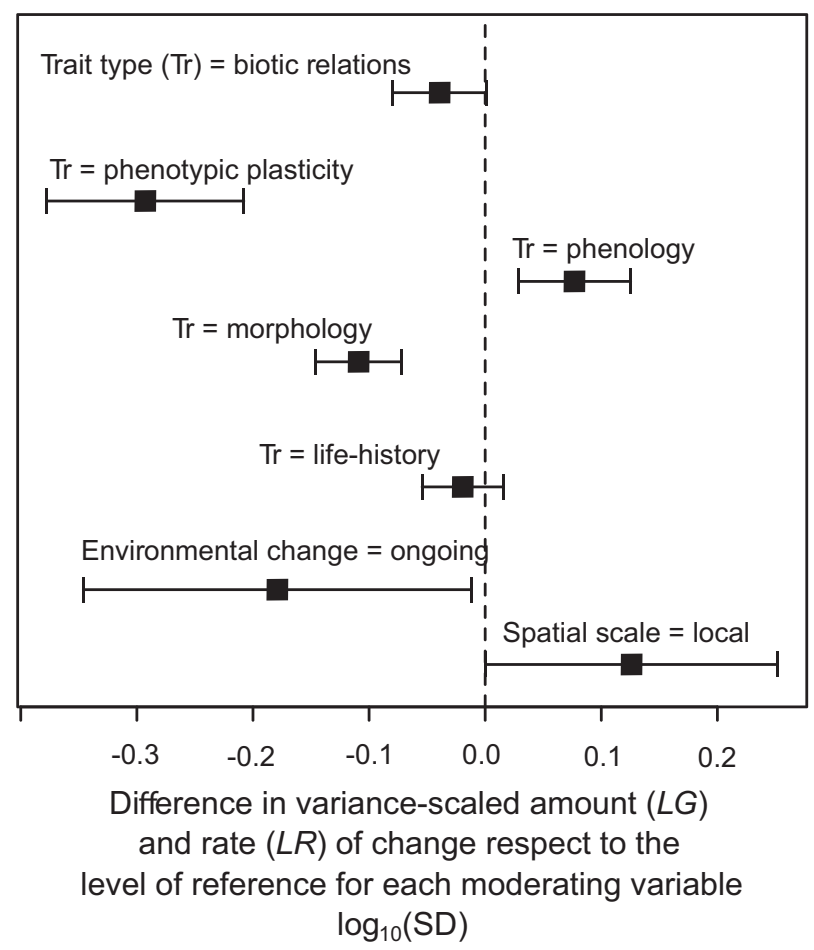

Figure 6: Effect of the spatial scale, environmental change, and trait type $(\mathrm{Tr})$ on the variance-scaled amount and rate of change. The dashed line represents the reference level for each moderating variable. Estimates and 95\% confidence intervals according to model $1 \mathrm{~h} . \mathrm{b}$ are detailed in table S1. "Spatial scale = local" shows the difference between local and nonlocal (regional plus continental) scale. "Environmental change $=$ ongoing" shows the difference between ongoing environmental change and novel environmental change. Trait type shows the difference between each trait type and physiological traits (dashed line). 
et al. 2008; Crispo et al. 2010). Moreover, the pattern cannot be explained purely by phenotypic plasticity because the transgenerational phenotypic change follows the same abrupt change pattern, even when the annuals in longitudinal design are analyzed separately, which arguably captures microevolutionary change.

Some methodological issues could partially explain the overall pattern of quick initial change followed by stasis, including measurement error, biased selection of traits, biased selection toward studies in which greater changes are expected, and publication bias in the same direction (see Hendry et al. 2008). These issues cannot be completely ruled out, but even if they reduce the precision and/or accuracy of the estimation, they cannot explain why the greatest amounts of change were not found at the greatest elapsed times or why we found greater amounts of change at shorter elapsed times. Moreover, genetic change may be greatest immediately after a disturbance because this is when the population is farthest from its phenotypic optimum (Reznick 1997; Kinnison and Hendry 2001; Hendry et al. 2008). Particularly rapid changes may not be sustainable over long time periods due to very low absolute fitness or the depletion of genetic variation (Bürger and Lynch 1995; Boulding and Hay 2001; Kinnison and Hairston 2007). Finally, natural populations may experience strong selection over short time intervals, but the direction of selection may vary through time and therefore cancel out over longer time intervals (Gingerich 2001; Hendry and Kinnison 2001; Grant and Grant 2002; Estes and Arnold 2007). These processes may act together to produce the pattern of change observed here, characterized by high intrinsic rate of change occurring in the first few years and no directional change sustained over the elapsed time.

This general pattern of abrupt phenotypic change followed by stasis is consistent with the bounded pattern of evolution described by Uyeda et al. (2011) for timescales shorter than a million years: changes are constrained and do not accumulate over time. However, the model of strict stasis with a fixed optimum could not explain the significant changes observed (Voje et al. 2018). The observed pattern may be compatible with a model of fluctuating optimum where stasis is the result of populations tracking the bounded movements of an optimum (Voje et al. 2018). However, we think that such bounding is not given by the environmental conditions; rather, developmental and functional constraints (Arnold 1992) may define a broad adaptive zone (the boundaries) within which a narrower peak can shift tracking environmental changes.

Transgenerational change represents the heritable component of phenotypic change, so it is a proxy for evolution. According to this proxy, our empirical results show that the overall evolutionary path of angiosperms over timescales of $<300$ years is characterized by high intrinsic rate of change, and no directional change sustained over the elapsed time. This is similar to the patterns reported by Hendry et al. (2008) for animals. Only in the transgenerational phenotypic change in the herbaceous plants was there an increase in the variance-scaled amount of change over time, but the increase was very low and consistent with an intermediate pattern between stasis and unbiased random walk. Therefore, we argue that there is an initial abrupt directional change in response to the environmental novelty, followed by smaller, independent random changes once a new optimum has been reached. This would be a combination of two dynamics, more likely to be observed in herbaceous plants due to their faster turnover of individuals in the population. The first phase would fit an Ornstein-Uhlenbeck process (Lande 1976), as has been observed in, for example, a range expansion of reed warblers (Lo Cascio Sætre et al. 2017). The second phase would result from stochastic environmental changes in this new habitat, as have been documented for, for example, the Darwin's finches habitat on Daphne Major Island in the Galápagos (Grant and Grant 2002).

The difference between transgenerational and cisgenerational phenotypic change may be due to phenotypic plasticity. Our results show that phenotypic plasticity introduces an initial change, evidenced in the effect on the intrinsic rate of change (gray symbols in fig. $5 B-5 D$ ), which tends to be greater in the cisgenerational case. Substantial plastic phenotypic shifts may occur in a single generation, and the magnitude of such effects could remain relatively constant over longer time intervals, so phenotypic plasticity could be responsible for an abrupt response, with no genetic change (Trussell and Etter 2001). But here we showed that some heritable (transgenerational) change occurs in angiosperms over contemporary timescales. Adaptive plasticity may substantially reduce the fitness costs of environmental change and, consequently, aid population persistence and slow genetic change in the plastic trait (Price et al. 2003; Ghalambor et al. 2007). Therefore, while adaptive phenotypic plasticity could explain a lack of genetic change, it cannot explain the weak or null relation between a given amount of transgenerational phenotypic change and elapsed time or the negative relation between rate of change and elapsed time. The retraction pattern, which is observed only for the cisgenerational phenotypic change, also may be explained by phenotypic plasticity. When a population faces a novel environment that is out of the previously experienced range, it may change plastically in an adaptive or maladaptive way (Ghalambor et al. 2007). If the response is maladaptive, it will strengthen selection and genetic evolution as a consequence (Grether 2005; Ghalambor et al. 2015). Therefore, the negative slope observed in the relation between the amount of cisgenerational phenotypic change and elapsed time may be due to early partially maladaptive 
plasticity that is later removed by genetic compensation (Grether 2005). Another possible explanation for the retraction pattern is that the environmental change leads to a genetic adaptive change with an initial relatively narrow variability. Later, in the new optimum, phenotypic variability may increase due to new genetic combinations, which could arrive by migration. Because the variance-scaled amount of change (LG) is inversely proportional to the pooled variance, this retraction-expansion of variance could produce the retraction pattern even if the mean value of the trait does not change after the initial shift.

Some of our results contradict common ideas about the links between generation time and evolutionary rate. We found that the intrinsic rate of transgenerational phenotypic change in woody plants is not smaller than that of herbaceous plants. On the contrary, it seems to be greater despite the fact that we used an absolute temporal scale (years instead of generations). Also, the trajectories of LG, LR, $|\log R R|$, and $\log _{10}(|d w|)$ through time are similar for woody and herbaceous plants. While variance-scaled amount and rate of change tend to be greater in woody species than in herbaceous ones, mean-scaled measurements show the opposite trend (table S1). For evolution over short periods of time, the upper boundary for evolutionary response per generation is set by standing genetic diversity. Woody plants have more within-population genetic diversity and a higher proportion of polymorphic loci than herbaceous plants (Hamrick and Godt 1996; Nybom 2004), which could enable fast evolution by selection (Petit and Hampe 2006). In addition, some annual species may escape from a stressful environment by adjusting their phenology (e.g., surviving unfavorable periods in the form of seeds), with other traits remaining unchanged. However, woody species are underrepresented in this database (12 over 153 species), and for this group the minimum elapsed time is 24 years. Additional methodological issues could partially explain the observed pattern in the instantaneous rate of change in both woody and herbaceous plants. First, because transgenerational studies worked with plants grown from seeds, any environmental novelty that had affected reproductive success (e.g., fecundity, fertility, pollen viability, pollination) may have changed the population of seeds in the first years even if its effects on the survival or vegetative traits of the resident individuals are not yet evident. Second, most of the common-conditions experiments were performed with the first filial generation, so maternal or epigenetic effects cannot be completely ruled out.

We found that life-history traits have an intermediate amount and rate of change, both variance scaled and mean scaled, with values similar to those of morphological and physiological traits. Moreover, the variance-scaled amount of change tends to be larger for life-history traits than for morphological ones. This was to be expected according to the evolvability patterns documented by Opedal (2019) and the selection pattern found by Kingsolver et al. (2001). That is, life-history traits (according our nomenclature) showed an intermediate evolvability similar to those of physiological traits, a tendency to have larger evolvabilities than morphological ones, and a directional selection similar to other traits. Plasticity traits showed the greatest amount and rate of mean-scaled change, but this is due to greater population variability, so they display the least variance-scaled amount and rate of change. Phenological traits showed an intermediate amount of change, albeit displaying a mean-scaled rate of change as fast as plasticity, and the greatest variancescaled rate of change. They thus appear to have high evolvability but also high heritability and to be under strong directional selection.

The lognormal distribution of the absolute rates of change is similar to those previously reported in the literature (Bone and Farres 2001; Hendry and Kinnison 2001). A still-open question is whether this is due to a limited capacity to change in the population or to the fact that small changes are enough to restore fitness. We do not know the strength of natural selection related to the changes recorded in this database; however, it seems that in nature directional selection follows a similar distribution (Kingsolver et al. 2001; Hereford et al. 2004; Kingsolver and Pfennig 2007; Kingsolver and Diamond 2011).

Over contemporary time, gene flow has the potential to be a larger source of genetic variation than mutation but can also erode local differentiation, leading to "ephemeral divergence" (Futuyma 2010). The effect of gene flow on the divergence between populations may also depend on the traits measured (Fitzpatrick et al. 2017). Our results show that at local scale, where gene flow between populations is probably more intense than between populations at regional or continental scales, the amount (LG) and rate of divergence between populations (LR and $\left.\log _{10}(|d w|)\right)$ were greater. In our database, the nonlocal scales are mostly related to invasion and range expansion processes, which likely produce demographic and genetic bottlenecks. In a local population experiencing an environmental novelty and surrounded by a regional population that does not experience it, continuous immigration will rescue the local population and may provide a more diverse substrate for natural selection (Garant et al. 2007).

In all of the models fitted to our database - even the most complete and best-fitted ones - the amount of residual heterogeneity is greater than expected by chance (table $S 1, Q_{E}$ test). This means that there are important moderating variables that have not been captured by our analysis. Variables related to the intensity of the natural selection, such as a categorization of the environmental novelties according to several types of change (e.g., pollution, range expansion, resource availability change), could be included as moderating variables. Finally, other biological and ecological properties of the 
species that seem to be related to rate of mutation and genetic diversity and that were not included here are plant height (Lanfear et al. 2013), breeding system, seed dispersal mode, and geographic range (Hamrick and Godt 1996).

The abrupt pattern of phenotypic change reported here has important consequences for management and conservation. The broad range of phenotypic responses illustrates the high adaptive potential of plant populations. However, on the basis of our findings, under a persistent modification in the environment a plant population should be expected to change in a very short time and then dramatically decrease its pace of change. In other words, if an adverse environmental novelty is not dealt with by a population in the first few years (by evolution or plastic response), this population may be at risk because an adaptive jump later on is unpredictable and typically takes many generations (Newman et al. 1985; Elena et al. 1996; Uyeda et al. 2011). Therefore, in practical terms, if a plant population does not maintain seed production and recruitment in the first few years following an environmental change, a future recovery should not be expected, and it may be necessary to intervene.

\section{Conclusions}

Our results show that angiosperms are capable of substantial amounts of phenotypic change over very short times, and no evidence of restrictions were found for those traits closely related to fitness or for long-lived species. Although phenotypic plasticity may increase the initial response, there is an important heritable component in such phenotypic change. Greater amounts and rates of phenotypic change found at local relative to nonlocal scale and the observed pattern of abrupt change followed by stasis suggest that standing variability is crucial for adaptation to environmental novelties and that new adaptive variability is unlikely to arise over contemporary timescales.

\section{Acknowledgments}

This study was supported by Fondo para la Investigación Científica y Tecnológica (FONCyT), Consejo Nacional de Investigaciones Científicas y Técnicas (CONICET; PIP 11220130100103), Secretaría de Ciencia y Tecnología (SECyT)Universidad Nacional de Córdoba (33620180100767CB and PRIMAR Res 248/18), the Inter-American Institute for Global Change Research (IAI; SGP-HW 090), and the Newton Fund. L.D.G. was supported by a CONICET and SECyT-Universidad Nacional de Córdoba postgraduate scholarship. We thank Oscar Humberto Bustos for insightful advice in mathematics and simulations. We thank A. A. Winn, C. M. Caruso, and three anonymous reviewers for valuable comments that helped improve the manuscript.
Statement of authorship: L.D.G. collected the data, performed the meta-analysis, ran the simulations, and interpreted the results; L.D.G. and S.D. wrote the manuscript.

\section{Data and Code Availability}

The new version of the database from the literature survey is stored in the Zenodo repository (https://zenodo.org) under the name "Gorné LD, Díaz S. 2019. Contemporary phenotypic change in plant quantitative traits" (https://doi.org /10.5281/zenodo.3251128).

\section{Literature Cited}

Arnold, S. J. 1992. Constraints on phenotypic evolution. American Naturalist 140:S85-S107.

Bevington, P. R., and D. K. Robinson. 2003. Data reduction and error analysis for the physical sciences. 3rd ed. McGraw-Hill, New York.

Bone, E., and A. Farres. 2001. Trends and rates of microevolution in plants. Genetica 112/113:165-182.

Boulding, E. G., and T. Hay. 2001. Genetic and demographic parameters determining population persistence after a discrete change in the environment. Heredity 86:313-324.

Bürger, R., and M. Lynch. 1995. Evolution and extinction in a changing environment: a quantitative-genetic analysis. Evolution 49:151163.

Campbell, O. W., and M. J. Donoghue. 2005. Phylomatic: tree assembly for applied phylogenetics. Molecular Ecology Notes 5:181-183.

Cooper, H., L. V. Hedges, and J. C. Valentine. 2009. The handbook of research synthesis and meta-analysis. 2nd ed. Russell Sage, New York.

Crispo, E., J. D. Dibattista, C. Correa, X. Thibert-Plante, A. E. Mckellar, A. K. Schwartz, D. Berner, et al. 2010. The evolution of phenotypic plasticity in response to anthropogenic disturbance. Evolutionary Ecology Research 12:47-66.

DeCoster, J. 2004. Meta-analysis notes. Accessed December, 21, 2013. http://www.stat-help.com/notes.html.

Elena, S. F., V. S. Cooper, and R. E. Lenski. 1996. Punctuated evolution caused by selection of rare beneficial mutations. Science 272:18021804.

Estes, S., and S. J. Arnold. 2007. Resolving the paradox of stasis: models with stabilizing selection explain evolutionary divergence on all timescales. American Naturalist 169:227-244.

Fisher, R. A. 1930. The genetical theory of natural selection. Oxford University Press, Oxford.

Fitch, W. M., and F. J. Ayala. 1994. Tempo and mode in evolution. Proceedings of the National Academy of Sciences of the USA 91:6717-6720.

Fitzpatrick, S. W., C. A. Handelsman, J. Torres-Dowdall, E. W. Ruell, E. D. Broder, J. A. Kronenberger, D. N. Reznick, et al. 2017. Gene flow constrains and facilitates genetically based divergence in quantitative traits. Copeia 105:462-474.

Futuyma, D. J. 2010. Evolutionary constraint and ecological consequences. Evolution 64:1865-1884.

Garant, D., S. E. Forde, and A. P. Hendry. 2007. The multifarious effects of dispersal and gene flow on contemporary adaptation. Functional Ecology 21:434-443. 
Ghalambor, C. K., K. L. Hoke, E. W. Ruell, E. K. Fischer, D. N. Reznick, and K. A. Hughes. 2015. Non-adaptive plasticity potentiates rapid adaptive evolution of gene expression in nature. Nature 525:372-375

Ghalambor, C. K., J. K. McKay, S. P. Carroll, and D. N. Reznick. 2007. Adaptive versus non-adaptive phenotypic plasticity and the potential for contemporary adaptation in new environments. Functional Ecology 21:394-407.

Gingerich, P. D. 1983. Rates of evolution: effects of time and temporal scaling. Science 222:159-161.

1993. Quantification and comparison of evolutionary rates. American Journal of Science 293A:453-478.

-2001. Rates of evolution on the time scale of the evolutionary process. Genetica 112/113:127-144.

. 2009. Rates of evolution. Annual Review of Ecology, Evolution, and Systematics 40:657-675.

Gorné, L. D., and S. Díaz. 2017a. A novel meta-analytical approach to improve systematic review of rates and patterns of microevolution. Ecology and Evolution 7:5821-5832.

. 2017b. Phenotypic microevolution in plant quantitative traits [data set, version 1.1]. Data from: A novel meta-analytical approach to improve systematic review of rates and patterns of microevolution. Ecology and Evolution 7:5821-5832, Zenodo, http://doi.org/10.5281 /zenodo.580095.

. 2019. Contemporary phenotypic change in plant quantitative traits [data set, version 1.2]. Data from: Meta-analysis shows that rapid phenotypic change in angiosperms in response to environmental change is followed by stasis. American Naturalist, Zenodo, http://doi.org/10.5281/zenodo.3251128.

Grant, P. R., and B. R. Grant. 2002. Unpredictable evolution in a 30-year study of Darwin's finches. Science 296:707-711.

Grether, G. F. 2005. Environmental change, phenotypic plasticity, and genetic compensation. American Naturalist 166:115-123.

Haldane, J. B. S. 1949. Suggestion as to quantitative measurement of rates of evolution. Evolution 3:51-56.

Hamrick, J. L., and M. J. W. Godt. 1996. Effects of life history traits on genetic diversity in plant species. Philosophical Transactions of the Royal Society B 351:1291-1298.

Hansen, T. F., C. Pélabon, and D. Houle. 2011. Heritability is not evolvability. Evolutionary Biology 38:258-277.

Hedges, L. V. 1981. Distribution theory for Glass's estimator of effect size and related estimators. Journal of Educational Statistics 6:107128.

. 1982. Statistical methods for meta-analysis. Statistical methodology in meta-analysis. National Institute of Education, Washington, DC.

Hedges, L. V., J. Gurevitch, and P. S. Curtis. 1999. The meta-analysis of response ratios in experimental ecology. Ecology 80:1150-1156.

Hendry, A. P., T. J. Farrugia, and M. T. Kinnison. 2008. Human influences on rates of phenotypic change in wild animal populations. Molecular Ecology 17:20-29.

Hendry, A. P., and M. T. Kinnison. 1999. The pace of modern life: measuring rates of contemporary microevolution. Evolution 53:1637-1653

. 2001. An introduction to microevolution: rate, pattern, process. Genetica 112/113:1-8.

Hereford, J., T. Hansen, and D. Houle. 2004. Comparing strengths of directional selection: how strong is strong? Evolution 58:21332143.

Houle, D. 1992. Comparing evolvability and variability of quantitative traits. Genetics 130:195-204.
Hunt, G. 2006. Fitting and comparing models of phyletic evolution: random walks and beyond. Paleobiology 32:578-601.

2012. Measuring rates of phenotypic evolution and the inseparability of tempo and mode. Paleobiology 38:351-373.

IPCC (Intergovernmental Panel on Climate Change). 2014. Climate change 2014: synthesis report. Contribution of Working Groups I, II and III to the Fifth Assessment Report of the Intergovernmental Panel on Climate Change. IPCC, Geneva.

. 2018. Global Warming of $1.5^{\circ} \mathrm{C}$ : an IPCC special report on the impacts of global warming of $1.5^{\circ} \mathrm{C}$ above pre-industrial levels and related global greenhouse gas emission pathways, in the context of strengthening the global response to the threat of climate change. IPCC, Geneva.

Jerling, L. 1985. Are plants and animals alike? a note on evolutionary plant population ecology. Oikos 45:150-153.

Kauffman, S., and S. Levin. 1987. Towards a general theory of adaptive walks on rugged landscapes. Journal of Theoretical Biology 128:11-45.

Keck, F., F. Rimet, A. Bouchez, and A. Franc. 2016. phylosignal: an R package to measure, test, and explore the phylogenetic signal. Ecology and Evolution 6:2774-2780.

Kingsolver, J. G., and S. E. Diamond. 2011. Phenotypic selection in natural populations: what limits directional selection? American Naturalist 177:346-357.

Kingsolver, J. G., H. E. Hoekstra, J. M. Hoekstra, D. Berrigan, S. N. Vignieri, C. E. Hill, A. Hoang, et al. 2001. The strength of phenotypic selection in natural populations. American Naturalist 157:245-261.

Kingsolver, J. G., and D. W. Pfennig. 2007. Patterns and power of phenotypic selection in nature. BioScience 57:561-572.

Kinnison, M. T., and N. G. Hairston. 2007. Eco-evolutionary conservation biology: contemporary evolution and the dynamics of persistence. Functional Ecology 21:444-454.

Kinnison, M. T., and A. P. Hendry. 2001. The pace of modern life II: from rates of contemporary microevolution to pattern and process. Genetica 112/113:145-164.

Lande, R. 1976. Natural selection and random genetic drift in phenotypic evolution. Evolution 30:314-334.

Lanfear, R., S. Y. W. Ho, T. Jonathan Davies, A. T. Moles, L. Aarssen, N. G. Swenson, L. Warman, et al. 2013. Taller plants have lower rates of molecular evolution. Nature Communications 4:1879.

Lo Cascio Sætre, C., C. Coleiro, M. Austad, M. Gauci, G. P. Sætre, K. L. Voje, and F. Eroukhmanoff. 2017. Rapid adaptive phenotypic change following colonization of a newly restored habitat. Nature Communications 8:8-13.

Lush, J. L. 1947. Animal breeding plans. 3rd ed. Iowa State College Press, Ames.

Lynch, M. 1990. The rate of morphological evolution in mammals from the standpoint of the neutral expectation. American Naturalist 136:727-741.

Newman, C. M., J. E. Cohen, and C. Kipnis. 1985. Neo-darwinian evolution implies punctuated equilibria. Nature 315:400-401.

Nybom, H. 2004. Comparison of different nuclear DNA markers for estimating intraspecific genetic diversity in plants. Molecular Ecology 13:1143-1155.

Opedal, Ø. H. 2019. The evolvability of animal-pollinated flowers: towards predicting adaptation to novel pollinator communities. New Phytologist 221:1128-1135.

Petit, R. J., and A. Hampe. 2006. Some evolutionary consequences of being a tree. Annual Review of Ecology, Evolution, and Systematics 37:187-214. 
Price, T. D., A. Qvarnström, and D. E. Irwin. 2003. The role of phenotypic plasticity in driving genetic evolution. Proceedings of the Royal Society B 270:1433-1440.

R Core Team. 2018. R: a language and environment for statistical computing. R Foundation for Statistical Computing, Vienna.

Revell, L. J. 2010. Phylogenetic signal and linear regression on species data. Methods in Ecology and Evolution 1:319-329.

Reznick, D. N. 1997. Evaluation of the rate of evolution in natural populations of guppies (Poecilia reticulata). Science 275:1934-1937.

Trussell, G. C., and R. J. Etter. 2001. Integrating genetic and environmental forces that shape the evolution of geographic variation in a marine snail. Genetica 112/113:321-337.

Tsagris, M., C. Beneki, and H. Hassani. 2014. On the folded normal distribution. Mathematics 2:12-28.

Uyeda, J. C., T. F. Hansen, S. J. Arnold, and J. Pienaar. 2011. The million-year wait for macroevolutionary bursts. Proceedings of the National Academy of Sciences of the USA 108:15908-15913.
Viechtbauer, W. 2010. Conducting meta-analyses in R with the metafor package. Journal of Statistical Software 36:1-48.

Voje, K. L. 2016. Tempo does not correlate with mode in the fossil record. Evolution 70:2678-2689.

Voje, K. L., J. Starrfelt, and L. H. Liow. 2018. Model adequacy and microevolutionary explanations for stasis in the fossil record. American Naturalist 191:509-523.

Westley, P. A. H. 2011. What invasive species reveal about the rate and form of contemporary phenotypic change in nature. American Naturalist 177:469-509.

Zanne, A. E., D. C. Tank, W. K. Cornwell, J. M. Eastman, S. A. Smith, R. G. Fitzjohn, D. J. McGlinn, et al. 2014. Three keys to the radiation of angiosperms into freezing environments. Nature 506:89-92.

Associate Editor: Christina M. Caruso Editor: Alice A. Winn

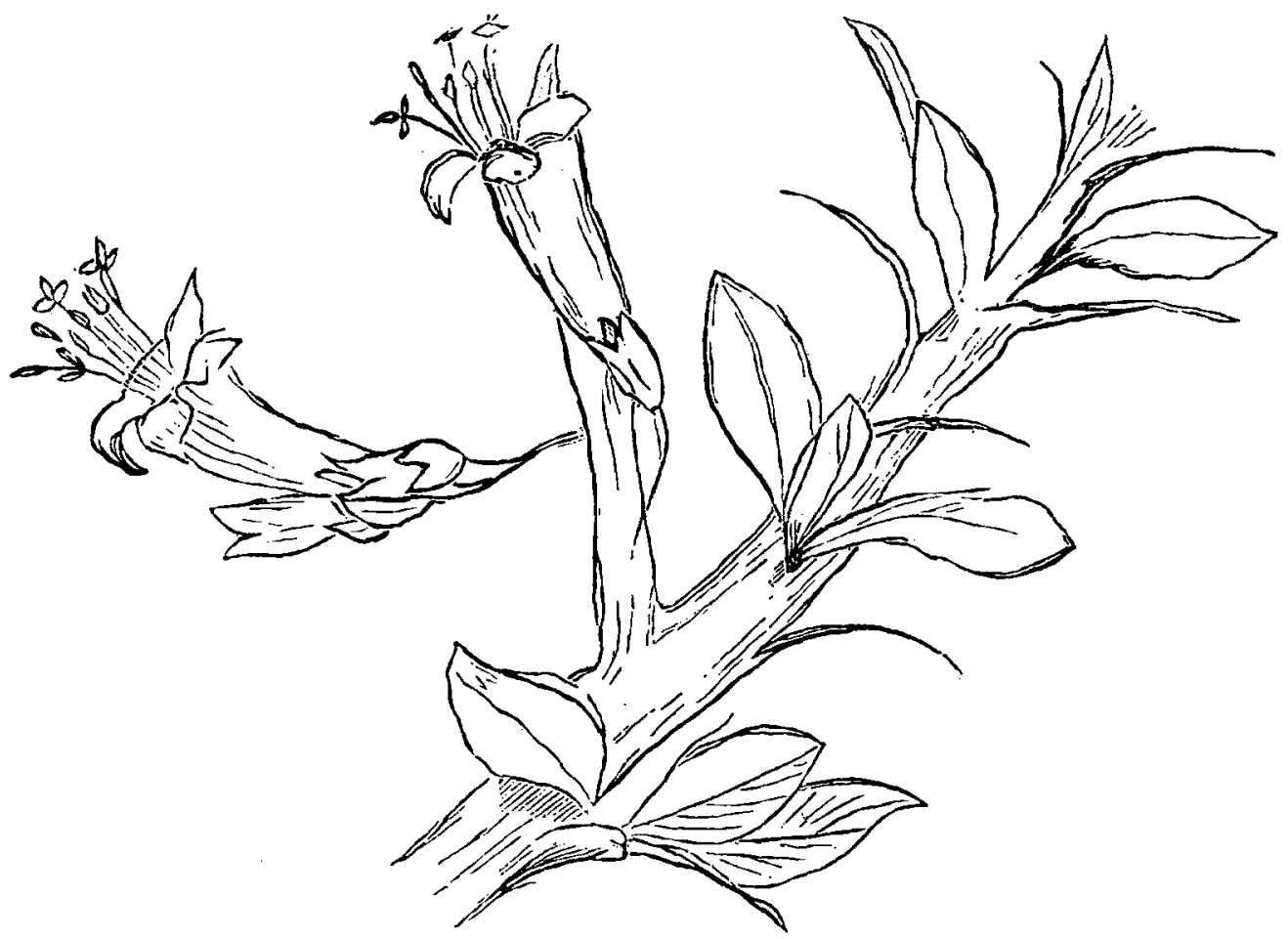

"Still another very common and at the same time a very curious plant is the Fouquiera splendens, one of the Tamariscineæ, and known to the Mexicans as 'ochotilla.' It grows all over the deserts of Arizona and among the rocks on the mountains. The branches are long and whiplike, armed with innumerable sharp, curved thorns an inch or more long. The flowers are of a bright scarlet, and form racemes at the ends of the branches." From "Botanical Notes from Tucson" by Jos. F. James (The American Naturalist, 1881, 15:978-987). 